\title{
A NOTE ON NAMES
}

In polite or formal speech, Russians address each other with name and patronymic, thus "Darya Fyodorovna" (Darya, daughter of Fyodor) or "Lev Nikolayevich" (Lev, son of Nikolai-the latter refers to Leo Tolstoy). At the other end of the spectrum, informal or intimate speech offers a myriad of variations on a single name: in the course of a single day, a person named "Sergey" might be called Seryozha, Seryozhka, Seryoga, Seryozhenka, and so on, with each variation carrying nuances of emotion, greater and lesser degrees of affection, intimacy, irritation, and more. The protagonists of Beyond Tula, Fyodor and Sergey, are constantly playing around with greater and lesser formality in address; since their conversations are the closest thing the novel has to a plot, the nuances are important. Generally speaking, the forms "Fedya" and "Seryozha" are casual and informal; the "-ka" suffix ("Fedka," "Seryozhka") contributes a playfully aggressive tone; further variations, like "Seryozhenka" or "Fedenka," are very tender. 